PROCEEDINGS OF THE

AMERICAN MATHEMATICAL SOCIETY

Volume 133, Number 8, Pages 2259-2266

S 0002-9939(05)07984-0

Article electronically published on March 4, 2005

\title{
POINTWISE UNIFORMLY ROTUND NORMS
}

\author{
JAN RYCHTÁř
}

(Communicated by Jonathan M. Borwein)

\begin{abstract}
It is shown that some properties of compact spaces $K$, such as carrying a strictly positive measure or being descriptive, are closely related to renormings of $C(K)$ or $C(K)^{*}$, respectively, by pointwise uniformly rotund norms.
\end{abstract}

Let $X$ be a Banach space. If $F$ is a closed, weak* dense subspace of $X^{*}$, then a norm $\|$.$\| on X$ is said to be $F$-uniformly rotund $\left(U R^{F}\right)$ if $\lim _{n \rightarrow \infty} f\left(x_{n}-y_{n}\right)=0$ for every $f \in F$ and every $x_{n}, y_{n} \in X$ such that

$$
\left\|x_{n}\right\|=\left\|y_{n}\right\|=1 \quad \text { and } \quad \lim _{n \rightarrow \infty}\left\|x_{n}+y_{n}\right\|=2 .
$$

The norm is called pointwise uniformly rotund $(p-U R)$ if it is $\mathrm{UR}^{F}$ for some weak* dense $F \subset X^{*}$ (see [20], [19]). In particular, the norm on $X=$ $Y^{*}$ is called weak $k^{*}$ uniformly rotund if it is $\mathrm{UR}^{Y}$ with the canonical embedding $Y \subset X^{*}=Y^{* *}$. The norm $\|$.$\| is called uniformly rotund in every direction$ $(U R E D)$ if $\lim _{n \rightarrow \infty}\left\|x_{n}-y_{n}\right\|=0$ for every $x_{n}, y_{n} \in X$ such that $\left\|x_{n}\right\|=\left\|y_{n}\right\|=$ $1, \lim _{n \rightarrow \infty}\left\|x_{n}+y_{n}\right\|=2$, and $x_{n}-y_{n} \in \operatorname{span}\left\{z_{0}\right\}$ for some $z_{0} \in X$.

A measure $\mu$ on a compact space $K$ is said to be strictly positive if $\mu(U)>0$ for every nonempty open set $U \subset K$. A compact space $K$ is called a uniform Eberlein compact if $K$ is homeomorphic to a weakly compact set in a Hilbert space [3]. A family $\mathfrak{N}$ of subsets of a compact space $K$ is said to be a network if every open set in $K$ is a union of members of $\mathfrak{N}$. A compact space $K$ is descriptive if there are closed sets $A_{n} \subset K$ and a network $\mathfrak{N}=\bigcup_{n} \mathfrak{N}_{n}$ such that every $\mathfrak{N}_{n}$ consists of relatively open and pairwise disjoint sets in $A_{n}$ [18, Lemma 3.1]. A compact space $(K, \tau)$ is fragmentable, if there is a metric $\varrho$ on $K$ such that for every $\varepsilon>0$ and every nonempty subset $M \subset K$ there exists an $\tau$-open set $\Omega \subset K$ such that $M \cap \Omega$ is nonempty and has $\varrho$-diameter less than $\varepsilon([7,[16])$. A Banach space $X$ is weakly compactly generated if there is a weakly compact set $K \subset X$ such that $X=\overline{\operatorname{span}} K$. For unexplained terms used in this paper we refer to 7] and [9.

Clearly, every p-UR norm is URED. URED norms are used in fixed point theory; see e.g. [5]. It turned out that p-UR norms can be used in characterizing some properties of compact spaces as follows.

Received by the editors March 25, 2003.

2000 Mathematics Subject Classification. Primary 46B03, 46B26, 46 E05.

Key words and phrases. Pointwise uniformly rotund norm, strictly positive measure, uniform Eberlein compacts, descriptive compacts, fragmentability.

This research was supported by NSERC 7926, FS Chia Ph.D. Scholarship for 2002/2003 and GAUK 277/2001, written as part of the author's Ph.D. thesis under the supervision of Professor N. Tomczak-Jaegermann and Professor V. Zizler.

(c) 2005 American Mathematical Society Reverts to public domain 28 years from publication 
Theorem 1. The space $C(K)$ of continuous functions on a compact space $K$ admits an equivalent pointwise uniformly rotund norm if and only if $K$ carries a strictly positive Radon probability.

Theorem 2. (a) For a compact space $K$, the space $C(K)^{*}$ admits a pointwise uniformly rotund (in general nondual) norm if and only if the space $L_{1}(\mu)$ is separable for every Radon probability on $K$.

(b) If $K$ is a descriptive compact space, then $C(K)^{*}$ admits an equivalent dual pointwise uniformly rotund norm.

(c) There is a nondescriptive (fragmentable) compact space $K$ such that $C(K)^{*}$ admits an equivalent dual pointwise uniformly rotund norm.

(d) If $K$ is a fragmentable compact space, then $C(K)^{*}$ admits an equivalent pointwise uniformly rotund norm. Consequently, the space $L_{1}(\mu)$ is separable for every Radon probability $\mu$ on a fragmentable compact $K$.

Theorem 3. Let $\mu$ be a finite measure. Then $L_{1}(\mu)$ admits an equivalent pointwise uniformly rotund norm if and only if $L_{1}(\mu)$ is separable.

Theorem 4. If a Banach space $X$ admits an equivalent pointwise uniformly rotund norm, then every weakly compact subset of $X$ is a uniform Eberlein compact.

For any finite measure $\mu$, the space $L_{1}(\mu)$ admits an equivalent URED norm by [13]; see also [5. Theorem 2.7.16]. Consequently, by Theorem 3, nonseparable $L_{1}(\mu)$ admits an equivalent URED norm and no p-UR norm. This is connected to [20, Problem 1]. Moreover, every weakly compact subset of $L_{1}(\mu)$ is a uniform Eberlein compact [1. Section 4]. Thus the converse of Theorem 4 does not hold even in WCG spaces. This is connected to [1, Problem 2.9].

There are fragmentable compact spaces such that $C(K)^{*}$ admits no dual strictly convex norm (e.g. $\left[0, \omega_{1}\right]$; see [5] Theorem 7.5.2]) and thus no dual p-UR norm (cf. Theorem 2(b) and (d)). It was proved in [21, Theorem 2] that $L_{1}(\mu)$ is separable for every Radon probability $\mu$ on a compact subset of the first Baire class. Thus split interval $S(I)$ is a nonfragmentable compact satisfying the conclusion of Theorem 2(d). By [13] and Kakutani's Theorem, $C(K)^{*}$ admits an equivalent URED norm for every compact $K$. The space $C\left([0,1]^{[0,1]}\right)^{*}$ does not admit an equivalent p-UR norm, as $L_{1}(\lambda)$ is nonseparable, where $\lambda$ is a product of Lebesgue measures on [0,1].

By [18], if $C(K)^{*}$ admits a dual weak* locally uniformly rotund norm, then $K$ is descriptive. Thus by Theorem 2(b), $C(K)^{*}$ admits an equivalent dual p-UR norm. By [7, Theorem 5.3.1], if $C(K)^{*}$ admits a dual strictly convex norm, then $K$ is fragmentable and thus, by Theorem 2 (d), $C(K)^{*}$ admits an equivalent p-UR norm. We do not know if in this case $C(K)^{*}$ admits an equivalent dual p-UR norm.

As shown in 14, there is a reflexive Banach space that does not admit any equivalent norm that is uniformly rotund in every direction. Thus this space does not admit any equivalent p-UR norm, although it admits an equivalent dual locally uniformly rotund norm.

Proof of Theorem 4. By Šmulyan's type theorem [5. Theorem 2.6.7], if the norm $\|\cdot\|$ on a Banach space $X$ is $\mathrm{UR}^{F}$, then the limit

$$
\lim _{t \rightarrow 0} \frac{\|f+t g\|^{*}-\|f\|^{*}}{t}
$$

exists for every $g \in X^{*},\|g\|^{*}=1$ and is uniform in $f \in F,\|f\|^{*}=1$, where $\|.\|^{*}$ is the dual norm to $\|$.$\| . In particular, the norm \|.\|^{*}$ is uniformly Gâteaux smooth on 
$F$. By [8], the dual unit ball $B_{F^{*}}$ is a uniform Eberlein compact in weak* topology of $F^{*}$. Hence, by [2], $F$ is a subspace of weakly compactly generated space $C\left(B_{F^{*}}\right)$.

For a given weak ${ }^{*}$ dense subspace $F \subset X^{*}$, let an operator $T: X \rightarrow F^{*}$ be given by $T=r \circ i$, where $i: X \rightarrow X^{* *}$ is the canonical inclusion and $r: X^{* *} \rightarrow F^{*}$ is the canonical restriction. The operator $T$ is one-to-one and $\sigma\left(X, X^{*}\right)-\sigma\left(F^{*}, F\right)$ continuous. Since $B_{F^{*}}$ is a uniform Eberlein compact in $\sigma\left(F^{*}, F\right)$ topology, $T(K)$ is a uniform Eberlein compact for every weakly compact set $K \subset X$. Hence $K$ is a uniform Eberlein compact, and the proof of Theorem 4 is finished.

Note that if $F$ admits a uniformly Gâteaux smooth norm, then $F^{*}$ admits a weak ${ }^{*}$ uniformly rotund norm (see [5, Theorem 2.6.7]), and thus the norm $\|\mid \cdot\| \|$ on $X$ defined by

$$
\|x\|^{2}=\|x\|^{2}+\|T x\|^{2}
$$

is an equivalent $\mathrm{UR}^{F}$ norm.

Proof of Theorem 1. Let $\mu$ be a strictly positive Radon probability measure on $K$. Then the identity map $I: C(K) \rightarrow L_{2}(\mu)$ is one-to-one and with a dense range. Thus the norm $\|\mid \cdot\|$ defined on $C(K)$ by

$$
\|f\|^{2}=\|f\|^{2}+\|I f\|_{L_{2}(\mu)}^{2}
$$

is an equivalent $\mathrm{UR}^{F}$ norm, where $F=\overline{\operatorname{span}} I^{*}\left(L_{2}(\mu)\right) \subset C(K)^{*}$.

Conversely, if $C(K)$ admits an equivalent $\mathrm{UR}^{F}$ norm, then $F$ is a subspace of a weakly compactly generated space (see the proof of Theorem 41). Thus $\ell_{1}(\Gamma)$ is not a subspace of $F$ for any uncountable set $\Gamma$; see [9, Chapter 11]. By [17, Lemma 1.3], there is a Radon probability $\mu$ on $K$ such that $F \subset L_{1}(\mu) \subset C(K)^{*}$. Note that the measure $\mu$ is strictly positive as $F$ is weak ${ }^{*}$ dense in $C(K)^{*}$. This concludes the proof of Theorem 1 .

Proof of Theorem 3. If $L_{1}(\mu)$ is separable, then it admits an equivalent p-UR norm with the same proof as that of 5, Corollary 2.6.9]. Assume that $L_{1}(\mu)$ is nonseparable and admits an equivalent $\mathrm{UR}^{F}$ norm. We claim that $F$ is norm separable. This means that $L_{1}(\mu)^{*}$ is weak* separable, which is a contradiction with 9 , Theorem 11.3].

To prove our claim, let us identify $L_{1}(\mu)^{*} \cong L_{\infty}(\mu)$ with $C(\Omega)$, where $\Omega$ is a Stonian space for measure $\mu$ (see [4, Appendix B] for details). Since the measure $\mu$ is finite, the space $L_{1}(\mu)^{*}$ admits an equivalent weak* uniformly rotund norm. By Theorem 1, $\Omega$ carries a strictly positive probability measure. In particular, $\Omega$ has a property ccc, that is, every collection of pairwise disjoint open sets of $\Omega$ is countable. Thus we only need to prove the following fact, which is a version of [17. Theorem 4.5(a) and Proposition 4.7].

Fact 5. Let $\Omega$ be a compact space with property ccc and let $X \subset C(\Omega)$ be isomorphic to a subspace of a weakly compactly generated space. Then $X$ is separable.

Proof. By 7, Theorem 7.2.2], there exists a Markushevich basis of $X$, i.e. a biorthogonal system $\left\{x_{\gamma}, f_{\gamma}\right\}_{\gamma \in \Gamma} \subset X \times X^{*}$ such that $\overline{\operatorname{span}}\left\{x_{\gamma} ; \gamma \in \Gamma\right\}=X$ and $\left\{f_{\gamma} ; \gamma \in \Gamma\right\}$ separates points of $X$. We may and do assume that $\left\|x_{\gamma}\right\|=3$. By [10], there exists a decomposition of $\Gamma=\bigcup_{n=1}^{\infty} \Gamma_{n}$ such that, for every $n \in \mathbb{N}$,

$$
\emptyset \neq{\overline{\left\{x_{\gamma} ; \gamma \in \Gamma_{n}\right\}}}^{\sigma\left(X^{* *}, X^{*}\right)} \backslash\left\{x_{\gamma} ; \gamma \in \Gamma_{n}\right\} \subset B_{X^{* *}} .
$$


Take $n$ such that $\Gamma_{n}$ is uncountable and define open sets $U_{\gamma} \subset \Omega$ by $U_{\gamma}=\{\omega \in$ $\left.\Omega ;\left|x_{\gamma}(\omega)\right|>2\right\}$ for $\gamma \in \Gamma_{n}$. Since $\Omega$ has ccc, there is a sequence $\left\{\gamma_{i}\right\}_{i=1}^{\infty} \subset \Gamma_{n}$ such that $\bigcap_{i=1}^{\infty} U_{\gamma_{i}} \neq \emptyset$ [17, Lemma 4.2]. Thus there is $\omega \in \bigcap_{i=1}^{\infty} U_{\gamma_{i}}$ such that $\left|x_{\gamma_{i}}(\omega)\right|>2$ for every $i \in \mathbb{N}$, a contradiction with (2). Thus $X$ is separable. This concludes the proof of Fact 5 and the proof of Theorem 3 is complete.

Remark. After submission, we learned that Theorem 3 was proved by a different method in [6, Theorem 2.11].

Proof of Theorem 2(a). Theorem follows easily from Theorem 3 and the Kakutani's Theorem; see e.g. [15].

Proof of Theorem 2(b). Let $\|\cdot\|_{1}$ be the canonical dual norm on $C(K)^{*}$. Fix the family $\mathfrak{N}=\bigcup_{n=1}^{\infty} \mathfrak{N}_{n}$ given by the definition of descriptivity of $K$. Consider $\mathfrak{N} \subset$

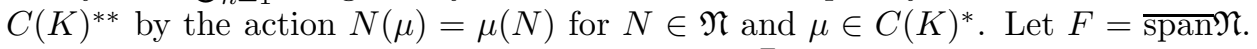
We will show that there is an equivalent dual $\mathrm{UR}^{F}$ norm on $C(K)^{*}$.

We claim that $F$ is weak* dense in $C(K)^{* *}$. Indeed, $\mu(G)=0$ for all open $G \subset K$ whenever $\mu(N)=0$ for all $N \in \mathfrak{N}$, as $\mathfrak{N}$ is a $\sigma$-isolated network consisting of relatively open pairwise disjoint sets.

Define a norm $\|$.$\| on C(K)^{*}$ in four steps, similarly as in [18, Proof of Theorem 3.3]. First, for every $n \in \mathbb{N}$, define a convex function $F_{n}$ on $C(K)^{*}$ by

$$
F_{n}(\mu)^{2}=\sum_{N \in \mathfrak{N}_{n}}|\mu|(N)^{2} .
$$

The function $F_{n}$ is weak* lower semi-continuous on $C\left(A_{n}\right)^{*}$. Second, for every $n, m \in \mathbb{N}$, define a weak* lower semi-continuous seminorm $\|\cdot\|_{m, n}$ on $C(K)^{*}$ by

$$
\|\mu\|_{m, n}^{2}=\inf \left\{\|\mu-u\|_{1}^{2}+m^{-1} F_{n}(u)^{2} ; u \in C\left(A_{n}\right)^{*}\right\} .
$$

Third, define an equivalent dual norm on $C(K)^{*}$ by

$$
\|\mu\|_{+}^{2}=\|\mu\|_{1}^{2}+\sum_{m, n \in \mathbb{N}} 2^{-m-n}\|\mu\|_{m, n}^{2} .
$$

\section{Claim 6.}

$$
\lim _{\omega \rightarrow \infty}\left(\mu_{\omega}-\nu_{\omega}\right)(N)=0,
$$

for all $n \in \mathbb{N}, N \in \mathfrak{N}_{n}$ and all positive measures $\mu_{\omega}, \nu_{\omega} \in C(K)^{*}, \omega \in \mathbb{N}$, such that $\left\|\mu_{\omega}\right\|_{1} \leq 1,\left\|\nu_{\omega}\right\|_{1} \leq 1$, and

$$
\lim _{\omega \rightarrow \infty} 2\left\|\mu_{\omega}\right\|_{+}^{2}+2\left\|\nu_{\omega}\right\|_{+}^{2}-\left\|\mu_{\omega}+\nu_{\omega}\right\|_{+}^{2}=0 .
$$

Once the claim is proved, finally define a norm $\|\cdot\|$ by

$$
\|\mu\|^{2}=\inf \left\{\left\|\mu_{1}\right\|_{+}^{2}+\left\|\mu_{2}\right\|_{+}^{2} ; \mu_{i} \in M(K), \mu_{i} \geq 0, \mu=\mu_{1}-\mu_{2}\right\} .
$$

Using the compactness argument, it follows from the weak* lower semicontinuity of $\|\cdot\|_{+}$that the infimum in (5) is attained for every $\mu \in C(K)^{*}$ and that the norm $\|\cdot\|$ is an equivalent dual norm on $C(K)^{*}$. Thus (3) holds whenever $\left\|\mu_{\omega}\right\|=1=$ $\left\|\nu_{\omega}\right\|$ and $\lim _{\omega \rightarrow \infty}\left\|\mu_{\omega}+\nu_{\omega}\right\|=2$. Hence the norm $\|$.$\| is \mathrm{UR}^{F}$. 
Proof of Claim [6] Fix $n \in \mathbb{N}$ and $N \in \mathfrak{N}_{n}$. From (44) and a convexity argument,

$$
\lim _{\omega \rightarrow \infty} 2\left\|\mu_{\omega}\right\|_{m, n}^{2}+2\left\|\nu_{\omega}\right\|_{m, n}^{2}-\left\|\mu_{\omega}+\nu_{\omega}\right\|_{m, n}^{2}=0,
$$

for every $m \in \mathbb{N}$. From a compactness argument, for every $\omega, m \in \mathbb{N}$, there are positive measures $u_{\omega}^{m, n}, v_{\omega}^{m, n} \in C\left(A_{n}\right)^{*}$ such that

$$
\begin{gathered}
\left\|\mu_{\omega}\right\|_{m, n}^{2}=\left\|\mu_{\omega}-u_{\omega}^{m, n}\right\|_{1}^{2}+m^{-1} F_{n}\left(u_{\omega}^{m, n}\right)^{2} \text { and } \\
\left\|\nu_{\omega}\right\|_{m, n}^{2}=\left\|\nu_{\omega}-v_{\omega}^{m, n}\right\|_{1}^{2}+m^{-1} F_{n}\left(v_{\omega}^{m, n}\right)^{2} .
\end{gathered}
$$

Consequently,

$$
F_{n}\left(u_{\omega}^{m, n}\right) \leq m\left\|\mu_{\omega}\right\|_{m, n} \leq m\left\|\mu_{\omega}\right\|_{1} \leq m
$$

and similarly $F_{n}\left(v_{\omega}^{m, n}\right) \leq m$. By passing to a subsequence, we may assume that

$$
\lim _{\omega \rightarrow \infty}\left\|\mu_{\omega}\right\|_{m, n}=d_{m, n}=\lim _{\omega \rightarrow \infty}\left\|\nu_{\omega}\right\|_{m, n} .
$$

The sequence $\left\{\|\mu\|_{m, n}\right\}_{m=1}^{\infty}$ is nonincreasing for every measure $\mu \in C(K)^{*}$. Thus there is $d_{n}=\lim _{m \rightarrow \infty} d_{m, n}$. Choose $\varepsilon>0$ and let $m_{0} \in \mathbb{N}$ be such that $d_{m_{0}, n}<$ $d_{n}+\varepsilon$. We will estimate $\left|\left(\mu_{\omega}-\nu_{\omega}\right)(N)\right|$ by

$$
\left|\left(\mu_{\omega}-u_{\omega}^{m_{0}, n}\right)(N)\right|+\left|\left(u_{\omega}^{m_{0}, n}-v_{\omega}^{m_{0}, n}\right)(N)\right|+\left|\left(v_{\omega}^{m_{0}, n}-\nu_{\omega}\right)(N)\right| .
$$

By a convexity argument and (6), (7), (8)),

$$
\lim _{\omega \rightarrow \infty} 2 F_{n}\left(u_{\omega}^{m_{0}, n}\right)^{2}+2 F_{n}\left(v_{\omega}^{m_{0}, n}\right)^{2}-F_{n}\left(u_{\omega}^{m_{0}, n}+v_{\omega}^{m_{0}, n}\right)^{2}=0 .
$$

Since $u_{\omega}^{m_{0}, n}$ and $v_{\omega}^{m_{0}, n}$ are positive measures, by a convexity argument again

$$
\lim _{\omega \rightarrow \infty}\left|\left(u_{\omega}^{m_{0}, n}-v_{\omega}^{m_{0}, n}\right)(N)\right|=0 .
$$

In order to estimate $\left|\left(\mu_{\omega}-u_{\omega}^{m_{0}, n}\right)(N)\right|$, consider a measure

$$
u=\mu_{\omega} \Upsilon_{N}+u_{\omega}^{m_{0}, r} \Upsilon_{K \backslash N}
$$

in the definition of $\left\|\mu_{\omega}\right\|_{m, n}$, where $\mu \uparrow_{A}$ means the restriction of $\mu$ on $A \subset K$. We get

$$
\begin{aligned}
\left\|\mu_{\omega}\right\|_{m, n}^{2} & \left.\leq\left\|\left.\left(\mu_{\omega}-u_{\omega}^{m_{0}, n}\right)\right|_{K \backslash N}\right\|_{1}^{2}+m^{-1} F_{n}\left(\mu_{\omega}\right\rceil_{N}+u_{\omega}^{m_{0}, n} \uparrow_{K \backslash N}\right)^{2} \\
& \left.\leq\left\|\left.\left(\mu_{\omega}-u_{\omega}^{m_{0}, n}\right)\right|_{K \backslash N}\right\|_{1}^{2}+m^{-1}\left(F_{n}\left(\mu_{\omega}\right\rceil_{N}\right)+F_{n}\left(u_{\omega}^{m_{0}, n} \uparrow_{K \backslash N}\right)\right)^{2} \\
& \leq\left\|\left.\left(\mu_{\omega}-u_{\omega}^{m_{0}, n}\right)\right|_{K \backslash N}\right\|_{1}^{2}+m^{-1}\left(\mu_{\omega}(N)+F_{n}\left(u_{\omega}^{m_{0}, n}\right)\right)^{2} \\
& \leq\left\|\left.\left(\mu_{\omega}-u_{\omega}^{m_{0}, n}\right)\right|_{K \backslash N}\right\|_{1}^{2}+m^{-1}\left(1+m_{0}\right)^{2} .
\end{aligned}
$$

Thus, for all $m \in \mathbb{N}$,

$$
\begin{aligned}
& \limsup _{\omega \rightarrow \infty}\left\|\left.\left(\mu_{\omega}-u_{\omega}^{m_{0}, n}\right)\right|_{K \backslash N}\right\|_{1}^{2} \geq \lim _{\omega \rightarrow \infty}\left\|\mu_{\omega}\right\|_{m, n}^{2}-m^{-1}\left(1+m_{0}\right)^{2}, \\
& \limsup _{\omega \rightarrow \infty}\left\|\left.\left(\mu_{\omega}-u_{\omega}^{m_{0}, n}\right)\right|_{K \backslash N}\right\|_{1}^{2} \geq d_{m, n}^{2}-m^{-1}\left(1+m_{0}\right)^{2}, \text { and } \\
& \limsup _{\omega \rightarrow \infty}\left\|\left.\left(\mu_{\omega}-u_{\omega}^{m_{0}, n}\right)\right|_{K \backslash N}\right\|_{1}^{2} \geq d_{n}^{2} .
\end{aligned}
$$

For all $\omega \in \mathbb{N}$ we have

$$
\begin{aligned}
\left|\left(\mu_{\omega}-u_{\omega}^{m_{0}, n}\right)(N)\right| & \leq \|\left(\mu_{\omega}-u_{\omega}^{m_{0}, n}\right)\left\lceil_{N} \|_{1}\right. \\
& =\left\|\mu_{\omega}-u_{\omega}^{m_{0}, n}\right\|_{1}-\left\|\left.\left(\mu_{\omega}-u_{\omega}^{m_{0}, n}\right)\right|_{K \backslash N}\right\|_{1} \\
& \leq\left\|\mu_{\omega}\right\|_{m_{0}, n}-\left\|\left.\left(\mu_{\omega}-u_{\omega}^{m_{0}, n}\right)\right|_{K \backslash N}\right\|_{1} .
\end{aligned}
$$


Thus

$$
\liminf _{\omega \rightarrow \infty}\left|\left(\mu_{\omega}-u_{\omega}^{m_{0}, n}\right)(N)\right| \leq d_{m_{0}, n}-d_{n} \leq \varepsilon .
$$

The same estimate holds for $\left|\left(\nu_{\omega}-v_{\omega}^{m_{0}, n}\right)(N)\right|$. The proof of Claim 6 is complete.

Proof of Theorem 2(c). First, we prove the following claim, which is a version of 11, Theorem 7.1].

Claim 7. Suppose that on a tree $T$ there is an increasing function $\varrho: T \rightarrow \mathbb{R}$ which is constant on no strictly increasing sequence in $T$. Then there is an equivalent dual p-UR norm on $C_{0}(T)^{*}$.

Proof of Claim 7 The space $C_{0}(T)^{*}$ can be identified with $\ell_{1}(T)$ with the canonical dual norm $\|\mu\|_{1}=\sum_{t \in T}|\mu(t)|$. Let us define $T^{+}$as the set of successors and $T_{0}$ as the set of all $t \in T^{+}$such that $\varrho(t)>\varrho\left(t^{-}\right)$. We may modify the function $\varrho$ so that it takes rational values at all points of $T_{0}$.

We will show that there is an equivalent dual $\mathrm{UR}^{F}$ norm where

$$
F=\overline{\operatorname{span}}\left\{\left\{\{s\} ; s \in T^{+}\right\} \cup\left\{[s, \infty) ; s \in T_{0}\right\} \cup\{T\}\right\} \subset \ell_{\infty}(T) .
$$

We claim that $F$ is weak ${ }^{*}$-dense in $C_{0}(T)^{* *}$. To prove it, let $\mu \in C(T)^{*}$ be such that $\mu(f)=0$ for all $f \in F$. We want to show that $\mu(\{t\})=0$ for all $t \in T$. Choose $t \in T$ and put $A(t)=\{u ; u \in(t, \infty), \varrho(u)=\varrho(t)\}$ and $B(t)=\min \{u \in$ $(t, \infty) ; \varrho(u)>\varrho(t)\}$. We have

$$
(t, \infty)=\bigcup_{u \in A(t)}\{u\} \cup \bigcup_{u \in B(t)}[u, \infty) .
$$

The union above is a union of disjoint open sets and $|\mu|$ is nonzero at most on countably many of them. Hence $\mu((t, \infty))=0$. Thus $\mu([t, \infty))=0$ for all $t \in T^{+}$. Since

$$
(0, t]=T \backslash \bigcup_{s \leq t}\left(\bigcup_{r \in s^{+} \backslash(0, t]}[r, \infty)\right),
$$

we have that $\mu((0, t])=0$ for all $t \in T$. Every limit element $t \in T$ is a limit of a sequence (of elements of $T_{0}$ ), thus $\mu((0, t))=0$ for all $t \in T$. Hence $\mu(\{t\})=0$ for all $t \in T$.

For every $q \in \mathbb{Q}$, the wedges $[s, \infty)$ with $s \in T_{0}$ and $\varrho(s)=q$ are disjoint, so we can define an equivalent dual norm on $C(T)^{*}$ by

$$
\|\mu\|_{+}^{2}=\|\mu\|_{1}^{2}+\sum_{s \in T^{+}}\left\|\mu \uparrow_{\{s\}}\right\|_{1}^{2}+\sum_{q \in \mathbb{Q}} c_{q}\left(\sum_{s \in T_{0} \cap \varrho^{-1}(q)}\left\|\mu \uparrow_{[s, \infty)}\right\|_{1}^{2}\right),
$$

where $c_{q}$ are some positive constants.

Let $\mu_{n}, \nu_{n} \in C_{0}(T)^{*}$ be positive elements such that $\left\|\mu_{n}\right\| \leq 1,\left\|\nu_{n}\right\| \leq 1$ and

$$
\lim _{n \rightarrow \infty} 2\left\|\mu_{n}\right\|_{+}^{2}+2\left\|\nu_{n}\right\|_{+}^{2}-\left\|\mu_{n}+\nu_{n}\right\|_{+}^{2}=0
$$

A standard convexity argument shows that

$$
\lim _{n \rightarrow \infty}\left(\mu_{n}-\nu_{n}\right)(T)=0, \lim _{n \rightarrow \infty}\left(\mu_{n}-\nu_{n}\right)(s)=0,
$$

for all $s \in T^{+}$, and

$$
\lim _{n \rightarrow \infty}\left(\mu_{n}-\nu_{n}\right)([s, \infty))=0,
$$

for any $s \in T_{0}$. Thus the norm $\|\cdot\|$ defined by (5) is $\mathrm{UR}^{F}$. This concludes the proof of Claim 7 . 
Now, let $\Lambda$ be a tree defined in [11 Section 10] and let $K$ be its Alexandroff compactification. Then $C(K)^{*}$ admits an equivalent dual p-UR norm by Claim 7 The space $C(K)^{*}$ does not admit any equivalent dual locally uniformly rotund norm, since $C(K)$ does not admit an equivalent Fréchet smooth norm [11, Corollary 10.9]. Thus $K$ is not a descriptive compact space by [18, Corollary 4.9]. The proof of Theorem 2 (c) is complete.

Proof of Theorem 2(d). Let $K$ be a fragmentable compact. By [7, Theorem 5.1.9 and Proof of Theorem 5.1.12(iii)], there is a family $\mathfrak{U}=\bigcup_{n=1}^{\infty} \mathfrak{U}_{n}$ of subsets of $K$ such that

(1) $\mathfrak{U}$ is a separating family, i.e. if $x \neq y \in K$, then there is $U \in \mathfrak{U}$ such that $\# U \cap\{x, y\}=1$

(2) $\mathfrak{U}$ is a network;

(3) for every $n \in \mathbb{N}, \mathfrak{U}_{n}$ is an open partitioning, i.e. $\mathfrak{U}_{n}=\left\{U_{\xi} ; \xi<\xi_{n}\right\}$ is well ordered such that $U_{\xi}$ is contained and is relatively open in $K \backslash\left(\bigcup_{\eta<\xi} U_{\eta}\right)$ for every $\xi<\xi_{n}$ and $K=\bigcup_{\xi<\xi_{n}} U_{\xi}$;

(4) for every $U \in \mathfrak{U}_{n+1}$ there is $V \subset \mathfrak{U}_{n}$ such that $\bar{U} \subset V$.

As $\mathfrak{U}_{n}$ is an open partitioning, it follows that

$$
\sum_{U \in \mathfrak{U}_{n}} \mu(U)=\mu(K)
$$

Define equivalent norms on $C(K)^{*}$

$$
\|\mu\|_{+}^{2}=|\mu|^{2}(K)+\sum_{n=1}^{\infty} 2^{-n} \sum_{U \in \mathfrak{U}_{n}}|\mu|^{2}(U)
$$

and

$$
\|\mu\|^{2}=\inf \left\{\left\|\mu_{1}\right\|_{+}^{2}+\left\|\mu_{2}\right\|_{+}^{2} ; \mu_{i} \in C(K)^{*}, \mu_{i} \geq 0, \mu=\mu_{1}-\mu_{2}\right\} .
$$

From a definition of a norm $\|\cdot\|_{+}$it follows that $\|\mu\|^{2}=\left\|\mu^{+}\right\|_{+}^{2}+\left\|\mu^{-}\right\|_{+}^{2}$. Let $F=\operatorname{span}\{U ; U \in \mathfrak{U}\} \subset C(K)^{* *}$. We will show that the norm $\|$.$\| is \operatorname{UR}^{F}$. Note that $F \subset C(K)^{* *}$ is weak* dense. Indeed, assume $\mu(U)=0$ for all $U \in \mathfrak{U}$ and let $G \subset K$ be an open set. Since $\mathfrak{U}$ is a network, we have $G=\bigcup_{n}\left(\bigcup \mathfrak{U}_{n}^{\prime}\right)$, where for every $n \in \mathbb{N}, \mathfrak{U}_{n}^{\prime}$ is a subfamily of $\mathfrak{U}_{n}$. Moreover, by condition (4), we may assume that $\mathfrak{U}_{n}^{\prime} \cap \mathfrak{U}_{m}^{\prime}=\emptyset$ for $m \neq n$. Thus

$$
\mu(G)=\mu\left(\bigcup_{n}\left(\bigcup \mathfrak{U}_{n}^{\prime}\right)\right)=\sum_{n} \mu\left(\bigcup \mathfrak{U}_{n}^{\prime}\right)=\sum_{n} \sum_{U \in \mathfrak{U}_{n}^{\prime}} \mu(U)=0,
$$

where the third equality hold as $\mathfrak{U}_{n}^{\prime}$ 's are relatively open partitioning. Thus, by a convexity argument, the norm $\|\cdot\|$ is $\mathrm{UR}^{F}$.

The proof of Theorem 2 (d) is complete.

\section{REFERENCES}

[1] S. Argyros, V. Farmaki: On the structure of weakly compact subsets of Hilbert spaces and application to the geometry of Banach spaces, Trans. Amer. Math. Soc. 289 (1985), 409-427. MR 0779073 (86h:46023)

[2] D. Amir and J. Lindenstrauss: The structure of weakly compact sets in Banach spaces, Ann. of Math. (2) 88 (1968), 35-46. MR0228983 (37:4562)

[3] Y. Benyamini and T. Starbird: Embedding weakly compact sets into Hilbert space, Israel J. Math. 23 (1976), no. 2, 137-141. MF0397372 (53:1231) 
[4] W. W. Comfort and S. Negrepontis: Chain conditions in Topology, Cambridge Tracts in Mathematics 79, Cambridge University Press, 1982. MR0665100 (84k:04002)

[5] R. Deville, G. Godefroy and V. Zizler: Smoothness and renormings in Banach spaces, Monographs and Surveys in Pure and Applied Mathematics 64, Pitman, 1993. MR1211634 (94d:46012)

[6] S. J. Dilworth, D. Kutzarova and S. L. Troyanski: On some uniform geometric properties in function spaces, General topology in Banach spaces, 127-135, Nova Sci. Publ., Huntington, NY, 2001. MR.1901540 (2003e:46035)

[7] M. Fabian: Gâteaux differentiability of convex functions and topology. Weak Asplund spaces. Canadian Mathematical Society Series of Monographs and Advanced Texts. A WileyInterscience Publication. John Wiley and Sons, Inc., New York, 1997 MF 1461271 (98h:46009)

[8] M. Fabian, G. Godefroy and V. Zizler: The structure of uniformly Gâteaux smooth Banach spaces, Israel J. Math. 124 (2001), 243-252. MR.1856517 (2002g:46015)

[9] M. Fabian, P. Habala, P. Hájek, V. Montesinos, J. Pelant and V. Zizler: Functional analysis and infinite dimensional geometry, Canadian Math. Soc. Books (Springer-Verlag), 2001. MR:1831176 (2002f:46001)

[10] M. Fabian, V. Montesinos and V. Zizler: Biorthogonal systems in weakly Lindelöf spaces, submitted.

[11] R. Haydon: Trees in renorming theory, Proc. London Math. Soc. (3) 78 (1999), 541-584. MR 1674838 (2000d:46011)

[12] K. John and V. Zizler: Smoothness and its equivalence in weakly compactly generated Banach spaces, J. Funct. Anal. 15 (1974), 161-166. MR0417759 (54:5807)

[13] D. N. Kutzarova: On an equivalent norm in $L_{1}$ which is uniformly convex in every direction, Constructive Theory of Functions, Sofia 84 (1984), 507-512.

[14] D. N. Kutzarova and S. L. Troyanski: Reflexive Banach spaces without equivalent norms which are uniformly convex or uniformly differentiable in every direction, Studia Math. 72 (1982), no. 1, 91-95. MR0665893 (83k:46024)

[15] H. E. Lacey: The isometric theory of classical Banach spaces, Die Grundlehren der mathematischen Wissenschaften, Band 208. Springer-Verlag, New York-Heidelberg, 1974. MR0493279 $(58: 12308)$

[16] I. Namioka: Fragmentability in Banach spaces. Interaction of topologies, Lecture Notes, Paseky School, Czech Republic (1999).

[17] H. P. Rosenthal: On injective Banach spaces and the spaces $L^{\infty}(\mu)$ for finite measures $\mu$, Acta Math. 124 (1970), 205-248. MR.0257721 (41:2370)

[18] M. Raja: Weak* locally uniformly rotund norms and descriptive compact spaces, J. of Functional Analysis 197 (2003), 1-13. MR1957673 (2004a:46009)

[19] J. Rychtáŕ: Renorming of $C(K)$ spaces, Proc. Amer. Math. Soc., 131 (2003), no. 7, 20632070 (electronic). MR1963751 (2004b:46018)

[20] M. A. Smith: Banach spaces that are uniformly rotund in weakly compact sets of directions, Can. J. Math. 29, No. 5 (1977), 963-970. MR0450942 (56:9232)

[21] S. Todorčević: Compact subsets of the first Baire class, J. Amer. Math. Soc. 12 (1999), no. 4, 1179-1212. MR1685782(2000d:54028)

Department of Mathematical and Statistical Sciences, University of Alberta, Edmonton, Alberta, Canada T6G 2G1

E-mail address: jrychtar@math.ualberta.ca

Current address: Department of Mathematical Sciences, University of North Carolina at Greensboro, Greensboro, North Carolina 27402

E-mail address: rychtar@uncg.edu 